\title{
Distortion of surfactant lamellar phases induced by surface roughness ${ }^{\star}$
}

\author{
Shirin Nouhi ${ }^{1 \mathrm{a}}$, Alexandros Koutsioubas ${ }^{2}$, Vassilios Kapaklis ${ }^{1}$, and \\ Adrian R. Rennie ${ }^{1, b}$ \\ ${ }^{1}$ Centre for Neutron Scattering, Uppsala University, 516, 75120 Uppsala, Sweden \\ 2 Jülich Centre for Neutron Science (JCNS) at Heinz Maier-Leibnitz Zentrum (MLZ), \\ Forschungszentrum Jülich GmbH, Lichtenbergstr. 1, 85748 Garching, Germany
}

Received 6 October 2019 / Accepted 6 August 2020

Published online 16 November 2020

\begin{abstract}
Self-assembly is a characteristic property of soft matter. Understanding the factors which assist or perturb this process is of great importance in many biological and industrial processes. Amphiphiles self-assemble and order into a variety of structures including well-ordered lamellar phases. The present work uses neutron reflectometry and neutron scattering to explore the effects of both interface roughness and temperature on the lamellar-phase structure of a non-ionic surfactant at a solid/liquid interface. The structure of concentrated solutions of tetraethyleneglycol dodecyl ether has been compared against a smooth surface and one with a roughness of the order of the lamellar spacing. This has been done in order to investigate the role perturbations have on the overall lamellar order, when these have length scales of the order of the interactions between neighboring lamellae. The results showed that the surfactant forms a well-ordered and aligned structure at a smooth surface, extending to a depth of several micrometers from the interface. Increasing the temperature of the sample and subsequent cooling promotes alignment and increases the number of oriented layers at the surface. The same sample forms a significantly less aligned structure against a rough surface that does not align to the same extent, even after heating. The perturbation of the structure caused by thermal fluctuations was found to be much less than that imposed by a small surface roughness.
\end{abstract}

\section{Introduction}

Amphiphiles with hydrophilic head groups and hydrophobic hydrocarbon tail regions occur widely as detergents, in biological membranes and in formulations for personal care and pharmaceutical products, coatings, etc. [1-4]. They self-assemble and order into a variety of structures such as micelles, liquid crystal phases, microemulsions

\footnotetext{
* Supplementary material in the form of one pdf file available from the Journal web page at https://doi.org/10.1140/epjst/e2020-900220-3

a Present address: SWERIM, Box 7047, SE-164 07 Kista, Sweden

b e-mail: Adrian.Rennie@physics.uu.se
} 
and other structures that display both long-range order and interesting dynamics. At solid/liquid interfaces, amphiphiles can organize themselves into well-ordered bilayer and even as multilayer structures. The structure and ordering of molecules at an interface are strongly influenced by both the physical and chemical properties [5,6]. Often simple ideas of the relative volumes of hydrophilic "head" groups and hydrophobic "tails" are used as guides to how surfactants will pack as planar or curved aggregates $[7,8]$. The induced ordering of amphiphiles by surfaces and interfaces has been discussed previously from both theoretical $[9,10]$ and experimental perspectives $[11,12]$, and this was found to be a key factor in determining their behavior and use in many potential applications [13]. For example, ordering in liquid crystal structures plays an important role in the biological function of cells, and disorder or defects can increase the risk of for example tissue cancer $[14,15]$.

There is considerable similarity between diblock copolymers and surfactants in that they both consist of two moieties that may tend to phase separate and can have quite different interactions with solvent molecules. Nanolithographic methods have been shown to direct self-assembly of diblock copolymers in terms of orientation and positional order $[16,17]$. In particular molecules with ethylene oxide head groups and alkyl tails that are often written as $\mathrm{C}_{\mathrm{n}} \mathrm{EO}_{\mathrm{m}}$, where $\mathrm{n}$ is the number of carbons in the tail and $\mathrm{m}$ is the number of ethylene oxide groups, are like very short copolymers. It has been shown that the temperature variation has a strong influence on the micelle formation temperature [18], adsorbed amount and the structure of alkyl ethoxy surfactants due to changes in hydration of the ethylene oxide groups. For example, their adsorption to sapphire surfaces at the critical micelle concentration, was found to increase significantly above the cloud temperature [19].

The structure formed by amphiphiles can be perturbed by temperature, physical and chemical properties of surfaces, and the addition of other components such as solvents. Understanding these different factors that induce or disturb the order of amphiphiles at interfaces is a major challenge, especially distinguishing between the effects when several perturbations are combined. Changes due to the addition of a new component such as spherical particles to the lamellar phase of $\mathrm{C}_{12} \mathrm{EO}_{5}$ have been described by Imai et al. and shown to influence the interlamellar interactions by imposing geometrical constraints on the packing of a planar structure [20-22]. The addition of colloidal particles suppressed the lamellar fluctuations, even at very low concentrations below 1 vol.\%, and caused the lamellar structures to change to micelles at higher concentrations above $\sim 3$ vol. $\%$.

Deformation or disorder in bilayers imposed either by internal bilayer interactions or by external factors such as guest components, can vary depending on the mechanical properties of these membranes. Previous studies have shown that mechanical properties of a membrane can be derived from the variation of scattered signals, providing insight into smectic penetration lengths [23]. These studies have been investigating the bulk order of lamellar structures, passing the probing beam through millimeter (s) thick samples. Other studies have been investigating interfacial structure of amphiphilic molecules on textured surfaces. It has been shown that lipid bilayers can assemble onto arrays of nanowires, of typically $100 \mathrm{~nm}$ diameter and length of a few $\mu \mathrm{m}$ following the topology of the nanowires over millimeter-sized areas [24]. Surfactant adsorption on surfaces with different roughness has been shown to occur with different kinetics and to equilibrate at different final amounts [25]. They found that the initial rate of adsorption for hexadecyl trimethyl ammonium bromide (CTAB), both below and above its critical micelle concentration, was higher on a rough surface, although the adsorption completed faster on the smooth surfaces. The structure of adsorbed CTAB was observed to be different on rough and smooth surfaces [26] with thicker and asymmetric bilayers that have a lower packing density and surface 
coverage formed on a rough surface. This raises the question: at which values of the surface roughness the corresponding perturbations become significant?

In the case of surfactant multilayers, a balance between the interactions in the bulk, those arising from stacking layers and with the solid surface determines the structure of lamellae. In the present study, we explore the influence of surface roughness on multilayer structures to gain understanding of the extent that small geometric perturbations, with length scales approximately the same as the lamellar spacing, influence the long-range ordering of surfactants. For this purpose, we have chosen a non-ionic surfactant, with known properties as a model system in a concentration range where ordered multilayer structures can form. The structure of the same surfactant dispersion is studied against a smooth and a rough surface at different temperatures, so that the effect of surface roughness can be distinguished from the perturbation due to thermal fluctuations.

\section{Neutron reflection and neutron scattering}

Neutron reflectometry is a surface sensitive technique for probing materials assembled at interfaces, and to the order of hundreds of micrometers into the bulk of the sample. In a typical neutron reflectometry experiment the intensity of the reflected beam is measured as a function of momentum transfer, $Q$, in the direction perpendicular to the interface $(z)$. The correlation length of the structure must be smaller than the coherent length of the beam in that direction to be probed using this technique. Normally this coherent length is about half a micron in the $z$ direction. In the present study three types of analysis have been performed to understand the ordering and alignment of the bilayer stacks. First, measuring and modeling the reflectivity, which is the ratio of the intensity of at the specular angle to that of the incoming beam. Under this condition, the average composition profile perpendicular to the surface is described as a model of different layers parallel to the interface. The scattered signal that occurs at other angles, provides structural information in other directions such as lateral inhomogeneity parallel to the surface and about lamellae that are not aligned with the surface. Secondly, by using a two-dimensional detector, it is possible to measure specular reflection and other scattering simultaneously. In a reflectometry experiment the resolution in the in-plane direction is low, hence only larger correlation length, typically several micrometers, can be probed. In this study, we used such data to analyze the full-width half maximum of the Bragg peak at the specular condition and for other orientations of lamellae in order to determine the size of ordered domains for these different structures that can occur at different depths from the interface. Further, measurements of the specular reflected signal at fixed incoming and outgoing beam angles were made as the sample is rotated. This type of rocking curve measurement provides information about the extent of lamellar alignment at the interface as diffraction occurs only when the Bragg condition is met.

Neutrons have a high penetration depth into some materials such as silicon, which makes them a useful tool to study the structures at solid/liquid or so-called buried interfaces. Neutrons are transmitted, refracted or reflected when at an interface. The interfaces relevant for neutrons are the boundaries between layers with different scattering length density. The scattering length density, $\rho$, of a material is given by:

$$
\rho=\Sigma n_{\mathrm{i}} b_{\mathrm{i}}
$$

where $n_{\mathrm{i}}$ is the number density for atoms of element $i$, and $b_{\mathrm{i}}$ is the scattering length. The scattering length represents the interaction potential of each element with neutrons and varies between different elements and isotopes. For example, neutrons have 
Table 1. Parameters used for modeling multilayer structure.

\begin{tabular}{|c|c|c|c|c|c|c|}
\hline Name & Formula & $\begin{array}{l}\text { Formula } \\
\text { Mass/ } \\
\mathrm{g} \mathrm{mol}^{-1} \\
\end{array}$ & $\begin{array}{l}\text { Density/ } \\
\mathrm{g} \mathrm{cm}^{-3}\end{array}$ & $\begin{array}{l}\text { Molecular } \\
\text { or formula } \\
\text { volume } / \AA^{\mathbf{3}}\end{array}$ & $\begin{array}{l}\text { Scattering } \\
\text { length } \\
\Sigma \mathbf{b} / \mathrm{fm}\end{array}$ & $\rho / 10^{-6} \AA^{-2}$ \\
\hline $\begin{array}{l}\text { Heavy } \\
\text { water }\end{array}$ & $\mathrm{D}_{2} \mathrm{O}$ & 20.03 & 1.105 & 30.0 & 19.1 & 6.35 \\
\hline Water & $\mathrm{H}_{2} \mathrm{O}$ & 18.02 & 0.9975 & 30.0 & -1.7 & -0.56 \\
\hline Silicon & $\mathrm{Si}$ & 28.08 & 2.33 & 12.9 & 4.14 & 2.07 \\
\hline Brij L4 & $\mathrm{C}_{12} \mathrm{H}_{25}\left(\mathrm{OCH}_{2} \mathrm{CH}_{2}\right)_{4} \mathrm{OH}$ & 362.54 & 0.95 & 633.7 & 4.89 & 0.10 \\
\hline $\begin{array}{l}\text { Brij L4 } \\
\text { tail }\end{array}$ & $\mathrm{C}_{12} \mathrm{H}_{25}$ & 169.33 & 0.75 & 374.9 & -13.72 & -0.40 \\
\hline $\begin{array}{l}\text { Brij L4 } \\
\text { head }\end{array}$ & $\left(\mathrm{OCH}_{2} \mathrm{CH}_{2}\right)_{4} \mathrm{OH}$ & 170.33 & 0.99 & 258.8 & 18.62 & 0.70 \\
\hline
\end{tabular}

very different scattering length densities for $\mathrm{H}_{2} \mathrm{O}$ and $\mathrm{D}_{2} \mathrm{O}$, see Table 1 for the values. Neutron reflectometry thus benefits from using $\mathrm{H}_{2} \mathrm{O}, \mathrm{D}_{2} \mathrm{O}$ or a mixture of $\mathrm{H}_{2} \mathrm{O}$ and $\mathrm{D}_{2} \mathrm{O}$ to change the contrast and enhance the signal from particular components. The refractive index, $n_{\mathrm{r}}$, for neutrons for a material is calculated from the scattering length density and wavelength, $\lambda$, as $n_{\mathrm{r}}=1-\left(\lambda^{2} \rho / 2 \pi\right)$.

In this study, neutron reflectometry was used to investigate the structure of the Brij L4 lamellar phase and the ordering of the lamellar structure close to rough and smooth surfaces. The reflectivity, $R(\mathbf{Q})$ is the ratio between the intensity of the reflected and incident beams at the specular condition, $\theta_{\mathrm{i}}=\theta_{\mathrm{f}}$, where $\theta_{\mathrm{i}}$ is the angle of the incident beam and $\theta_{\mathrm{f}}$ is the angle of the outgoing beam. At the specular condition, the momentum transfer $\mathbf{Q}$ is perpendicular to the interface, and the recorded signal provides information about the thickness, composition, and roughness of layers parallel to the interface. Reflectivity data at commonly shown against the magnitude of $\mathbf{Q}$ perpendicular to the interface and is given by:

$$
Q=(4 \pi / \lambda) \sin \left(\theta_{\mathrm{i}}\right)
$$

where $\lambda$ is the wavelength of the neutrons. The specular reflection has momentum transfer normal to the interface and so depends on the average scattering length density in layers parallel to a surface.

Scattering that is observed in other directions will provide information about other aspects of the structure such as that in the plane of the interface. At normal incidence a typical attenuation length for concentrated surfactants in $\mathrm{D}_{2} \mathrm{O}$ is about $1 \mathrm{~mm}$ and this is determined by the total cross-section for scattering and absorption. This is often dominated by the incoherent scattering from hydrogenous material. Importantly the depth that is probed in a scattering experiment can be varied by changing the angle of incidence [27]. For low angles of incidence, the consequent long path length may limit the depth that is investigated to about 0.01 to $0.1 \mathrm{~mm}$. Thus, in contrast to reflection that will explore depths limited by the coherence of the neutron beam or the structure at the interface, other low angle scattering will be averaged over the penetration depth of the beam. The combination of scattering observed away from the specular reflection and measurements of reflectivity therefore allows the structure to be investigated at different depths from an interface.

\section{Measurements and data treatment}

Neutron reflection experiments were performed on the MARIA reflectometer at MLZ, Munich $[28,29]$, which operates using a monochromatic beam and a vertical sample 
orientation. The resolution of MARIA is determined primarily by its $10 \%$ spread of wavelength. Reflectivity data were collected with two different wavelengths, having a small overlapping region. Reflectivity data for $Q<0.042 \AA^{-1}$ were collected with $\lambda=10 \AA$ and in the range $0.035 \AA^{-1}<Q<0.45 \AA^{-1}$ with $\lambda=5 \AA$.

Data reduction: The neutron signal on MARIA, is counted using a two-dimensional position sensitive detector, that allows the signal from both the specular reflection and the off-specular scattering to be recorded simultaneously. As described above, this allows interesting comparisons of interfacial ordering of lamellae and determining how far the oriented structure extends towards the bulk of the sample. However, it causes some challenges in the data reduction. Measurements consisted of coupled scans of the sample and detector, set in such way that the center of the detector meets the specular condition. Reflectivity values are obtained fitting a Gaussian peak at the center of the detector, additionally allowing for a background determination. However, for a strongly scattering sample such as our system, the Bragg diffraction peaks from the bulk, which can fall on either side of the region of interest of the detector for evaluation of the intensity of specular reflection depending on the sample alignment, contribute significantly to the background. Hence estimating the absolute reflectivity and subtracting the signal on the sides of the intense Bragg peaks becomes challenging (see Fig. S3, supporting information as an example). The effect becomes more pronounced when the Bragg peak is wider or there is more diffuse scattering due to random alignment of the surfactant structure, and much less pronounced when the Bragg peak is at the specular condition. The data recorded at all angles are subjected to the same reduction algorithm. However, since at low $Q\left(<0.05 \AA^{-1}\right)$ the signal to noise ratio is significantly smaller, the relative magnitude of the specular signal and the recorded noise does not allow a reliable discrimination of the reflectivity. Therefore, this region has been excluded from fitting of reflectivity data.

\section{Materials}

In the present study we used the non-ionic surfactant Brij L4, which has an average formula $\mathrm{C}_{12} \mathrm{H}_{25}\left(\mathrm{OCH}_{2} \mathrm{CH}_{2}\right)_{4} \mathrm{OH}$ (tetraethylene glycol dodecyl ether), or $\mathrm{C}_{12} \mathrm{EO}_{4}$, although there is some distribution of number of ethylene oxide moieties (Sigma Aldrich, P4391) and was diluted in water to concentrations of 40 and $55 \mathrm{vol} \%$. The sample at the higher concentration was very viscous and was stirred using a glass rod for about two minutes to make certain it was uniformly mixed. Previous studies reported the phase diagram of $\mathrm{C}_{12} \mathrm{EO}_{4}$ in $\mathrm{H}_{2} \mathrm{O}$ [30] and the phase diagram of shorter $\left(\mathrm{C}_{12} \mathrm{EO}_{3}\right)$ or longer $\left(\mathrm{C}_{12} \mathrm{EO}_{5}\right)$ surfactants $[31,32]$. Figure $\mathrm{S} 1$, in the supporting information, shows the phase diagram for $\mathrm{C}_{12} \mathrm{EO}_{4}$ in $\mathrm{H}_{2} \mathrm{O}$ from Mitchell et al., [30] with the region measured in this study marked in red. The exact phase boundaries that have been reported, with respect to temperature and concentration, differ slightly. However, they all agree that both 40 and 55 vol.\% Brij L4 is in the lamellar phase for the temperature range measured in these experiments.

Silicon crystals $5 \mathrm{~cm} \times 5 \mathrm{~cm} \times 1 \mathrm{~cm}$ cut with (111) planes on the large face were purchased from Crystran. One of the crystals was used with the polished smooth surface and the other crystal was etched and roughened in basic solution (pure Decon90) for 24 hours. The roughness of the crystals was checked with atomic force microscopy (see Fig. S2a, supporting information) and X-ray reflectivity measurements. Neutron reflectivity data (explained below) from the clean surface before injecting the samples are shown in Figure S2b, in supporting information. Both crystals were cleaned as described in our previous studies (see Ref. [33] for details). The cleaned crystals were characterized using neutron reflectivity with $\mathrm{D}_{2} \mathrm{O}, \mathrm{H}_{2} \mathrm{O}$ and a mixture of water that is neutron refractive index matched to silicon in order to determine the thickness of 
the oxide layer and roughness of the surface, in-situ prior to the sample injection. The smooth crystal surface was found to have an oxide $\left(\mathrm{SiO}_{2}\right)$ layer that was $\sim 10 \AA$ thick and with a roughness of $\sim 3 \AA$. The rough crystal was found to have a porous oxide layer with about $20 \%$ water in a layer of $60 \AA$ thickness with roughness of $\sim 30 \AA$.

\subsection{Reflectivity from Brij L4}

The reflectivity for a particular scattering length density profile can be calculated using the optical matrix method of Abelès [34,35]. Reflectivity data for the Brij lamellar structures were modelled using the byban program available on the web [36] which has been described also by Hellsing et al. [37]. The program allows the model of a repeating lamellar to be constrained by imposing stoichiometry for the head and tail groups of the surfactant molecule. The structural parameters used for modelling the reflectivity profile in this study were the thickness of the head, $t_{\mathrm{h}}$, and tail, $t_{\mathrm{t}}$, region of surfactant, the area per molecule, A, the interlayer distance occupied by solvent, $t_{\mathrm{s}}$, lamellar displacement due to thermal fluctuation, $\zeta$, and the number of layers, N. The slight difference between the present model and Hellsing et al. [37] is that we treated the bilayer as a four-layer structure with separate hydrophilic head and hydrophobic tail layers (see Fig. 1), whereas Hellsing et al. have used a simplified two-layer structure: one layer consists of surfactant including both heads and tails and a second solvent layer. We also had to vary the number of layers to model adequately the strong signal observed in some reflectivity curves. The number of layers in this program is an integer which was manually adjusted until the model could fit the intensity of the at least the first Bragg peak which was highly influenced by this parameter. Figure 1 shows a schematic diagram of the first few layers of the lamellar structure and the corresponding scattering length density profile calculated for 55 vol.\% Brij L4 at the $\mathrm{Si} / \mathrm{SiO}_{2} / \mathrm{D}_{2} \mathrm{O}$ interface. The total bilayer thickness, $d$, is calculated as:

$$
d=2 t_{\mathrm{h}}+t_{\mathrm{t}}+t_{\mathrm{s}}
$$

The intensity and width of the observed peaks depends primarily on the scattering length density contrast and the number of the repeating layers. The width of the observed peak is also strongly influenced by the instrumental resolution. Our analysis allows convolution of the instrumental resolution in $Q$ space with the model during the fitting process.

The lamellar structure never consists simply of a perfect multilayer of sheets of molecules parallel to the surface. The long-range order of the lamellar structure can be perturbed by different types of disorder that should be considered when modelling reflectivity data. One type of disorder occurs due to thermal motion that causes the layers to fluctuate slightly around their mean position. This type of fluctuation is different to the intermixing or roughness of adjacent layers which can be included in models using the methods described by Névot and Croce [38]. In the case of thermal fluctuations, the thickness and composition of different parts of the bilayer remain unchanged and a single bilayer fluctuates as a coherent unit (head and tail regions together) giving rise to local curvature of the layer. However, the fluctuation of different bilayers that are separated with a solvent layer, is not correlated and this decreases the intensity of the observed Bragg peaks. Thermal fluctuations can be in the same order as the lamellar spacing while for "roughness" to be physically meaningful, must be smaller than the thickness of layers and in the case of our model is constrained to be a fraction of the thickness that is not allowed to exceed 0.3.

The scattered intensity, $I(Q)$, depends on the shape and size of inhomogeneities or differences in scattering length density, as well as the correlations between these 


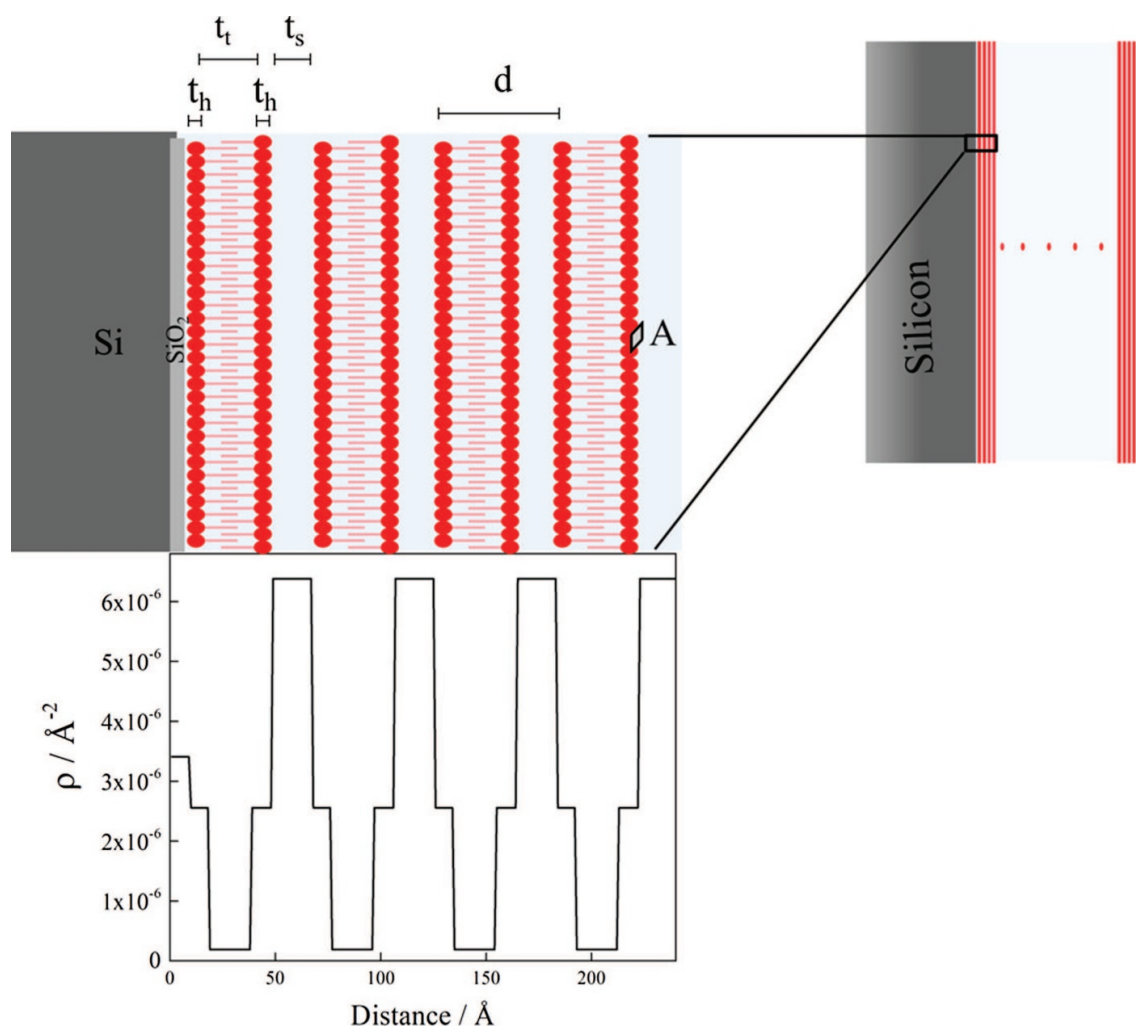

Fig. 1. Scattering length density profile of the lamellar structure supported on a silica surface. $t_{\mathrm{h}}$ refers to the thickness of the head group, $t_{\mathrm{t}}$ to the thickness of tail group, $t_{\mathrm{s}}$ to thickness of solvent layer, A is the area per molecule and d to the total bilayer thickness.

regions. Thermal disorder reduces the scattered intensity with an exponential dependence on the squared amplitude of the fluctuations, $\xi[39,40]$ :

$$
I(Q) \propto \exp (-2 w)
$$

where $2 w$ is known as the Debye-Waller factor that is defined as $Q^{2} \xi^{2} / 3$. Disorder due to bending fluctuations is pronounced in the surfactant lamellar phases and can be related to the thermal fluctuations described by the Debye-Waller factor. These fluctuations for a membrane can be calculated from the Caillé parameter [41], $\eta$ that depends on the bending modulus, $K$, and the compression modulus, $B$, of the lamellae and is given by:

$$
\eta=\frac{\pi k_{\mathrm{B}} T}{2 d^{2} \sqrt{K B}} .
$$

$k_{\mathrm{B}}$ is Boltzmann's constant, $T$ is the temperature, and $d$ is the repeat distance of the bilayer [42].

This parameter can be related directly to the amplitude of the thermal fluctuations by:

$$
\eta=\frac{\xi^{2} \pi^{2}}{d^{2}}
$$

where $\xi$ is the mean displacement from the central value [43]. These equations allow the mechanical response to be related to reflectivity and scattering data. In the 
present study, the mean displacement of the lamellae due to thermal fluctuations obtained from fitting reflectivity data was used to calculate the Caillé parameter and hence provide an estimate of the modulus.

Another type of disorder occurs due to small variations in the spacing of the layers and this decreases the intensity of the peaks very significantly. This type of disorder can be calculated for a structure with a variation in the lamellar spacing and this has been shown to decrease the intensity of Bragg peaks significantly. The reflectivity data measured for the samples in the present study show strong Bragg peaks including higher order diffraction and so the effect of this type of disorder is likely to be insignificant. During the analysis of the data, we tested a model gradient in composition of the bilayers at different depths from the interface. However, this resulted in a significant decrease in the intensity of the Bragg peaks, which could not be compensated by varying other parameters, which suggests that the effect of such disorder in the present system is insignificant.

As mentioned previously, reflectivity data provide structural information about the material forming structure parallel to the solid surface. Only a restricted number of parameters can be fitted unambiguously to reflectivity data, hence minimizing the number of freely variable parameters is a challenge that requires use of known constraints of composition and molecular structure. The present study work modeled the lamellar structure as a simple four-layer structure with a displacement which can arise from the combination of the various different types of disorder (mentioned above) and varies primarily with the temperature. Both the thermal fluctuations and misorientation induced by fixed structure at the interface influence the relative intensity of the different order Bragg peaks and also cause them to broaden.

In order to check independently the alignment of the lamellar sheets with the interface, the reflection was also measured while moving the sample over a small range of angles around the first order Bragg peak. This type of measurement is called a rocking curve where the full width half maximum (FWHM) of the intensity observed at the center of the detector gives an estimate of the alignment of the layers parallel to the interface.

Further analysis was performed by looking at the intensity distribution of the Bragg peaks at both specular and off-specular condition. For a perfect lamellar structure Bragg peaks with equal spacing are expected. The intensity of the peaks depends on the contrast and number of bilayers, and the width of the peaks depends on the crystal size (number of layers) and the instrumental resolution. Typically, in a reflectometry experiment the penetration depth of the beam depends on the attenuation coefficient and the incident angle of the beam, wavelength of neutrons and the critical angle of the interface [27] although the layers resolved at the interface would be limited by coherence as mentioned above. When the Bragg peaks are observed at the specular condition diffraction arises from crystal planes aligned parallel to the surface. The width provides information about the overall thickness of the aligned material at the surface. When diffraction is observed from other orientations, the Bragg peak appears at an off-specular condition and the depth probed depends on the penetration depth. The grain size is obtained for the crystallites forming parallel and non-parallel to the solid surface using the Debye-Scherrer formula $[40,44]$ :

$$
D=\frac{0.9 \lambda}{\beta \cos (\theta i)}
$$

where $D$ is the crystallite size and $\beta$ is the FWHM of the Bragg peak in radians. Note that the FWHM should be corrected for the resolution of the instrument. The resolution of MARIA is dominated by the $10 \%$ wavelength resolution, broadening the observed Bragg peaks and this has been included in the models. The domain size of the lamellae that may be oriented in other directions and are not parallel to the 
solid surface, was calculated using equation (7) from the width of the Bragg peaks when they are away from the specular condition.

\subsection{Sample holder}

The sample holder for this experiment has been described previously [45]. Briefly, in the set up for this experiment, the sample was sealed with a PTFE gasket between the silicon crystal and a polycarbonate base with injection and outlet ports. The temperature of the cell was controlled using a Julabo bath, circulating water through the aluminum parts that clamp the crystal and the base. The sample was injected into the cell at $20^{\circ} \mathrm{C}$ and the measured at $20,30,40,50,60^{\circ} \mathrm{C}$ and again at $20^{\circ} \mathrm{C}$ after exposure to the high temperature. This sequence of heating and subsequent cooling can be understood as allowing an observation of the influence of annealing at an elevated temperature. For each temperature rise, a pause of about $10 \mathrm{~min}$ was performed before data collection. On cooling, a longer interval of $30 \mathrm{~min}$ was allowed for thermal equilibration of the water circulating bath and sample.

\section{Results and discussion}

Figure 2 shows the reflectivity data and the model fits from 55 vol.\% Brij L4 at $20^{\circ} \mathrm{C}$ (after injecting the sample), on the left, and after heating and then cooling the sample back to $20^{\circ} \mathrm{C}$ on the right side. Fitting parameters obtained from the model fits for the sample at different temperatures at both surfaces are presented in Table S1, supporting information. The reflectivity data show several orders of Bragg peaks, which is an indication of many lamellae aligning themselves parallel to the surface. The sample exhibits very strong reflection, with the first order Bragg peak reaching more than $10 \%$ of the incident beam intensity, corresponding to a reflectivity $>0.1$. A self-organizing sample with such strong scattering provides a good model system to study the effect of different parameters on the distortion of the lamellar structure. A similar reflectivity magnitude has been observed previously from self-assembled micellar crystals such as those formed with Pluronic F127 (EO99$\mathrm{PO}_{56}-\mathrm{EO}_{99}$ ) polymeric surfactant in water [46].

Modelling the reflectivity data showed that surfactants self-assemble into multilayer structure with a bilayer thickness of $\sim 58 \AA$ aligning with the solid surface. This spacing corresponds to the spacing expected for 55 vol.\% Brij L4 in water and suggests that surface structure resembles the bulk. It is interesting to compare the present results with other systems, such as sodium aerosol-OT in water. The lamellar phase forms at low concentrations, about $10 \%$, because of the charge on the ionic groups and it forms multilayer structures at various interfaces $[47,48]$ at even lower concentrations. The spacing observed even at the lowest concentrations corresponded to $10 \%$ surfactant [49]. This has indicated that a surface pre-transition occurred in that system.

At $Q<0.08 \AA^{-1}$ there is a large contribution to the background due to diffraction from the bulk of the sample as mentioned above and this region was excluded from the fits. As expected, the observed intensity is larger than the calculated reflectivity because extra intensity is contributed from a distribution of crystallites in the bulk with less-defined orientation.

The same surfactant sample shows different reflectivity at the rough and smooth surfaces. The difference is very clear in the intensity of the first order Bragg peak which showed to be highly influenced by the repetition number of bilayers. At $20^{\circ} \mathrm{C}$ 


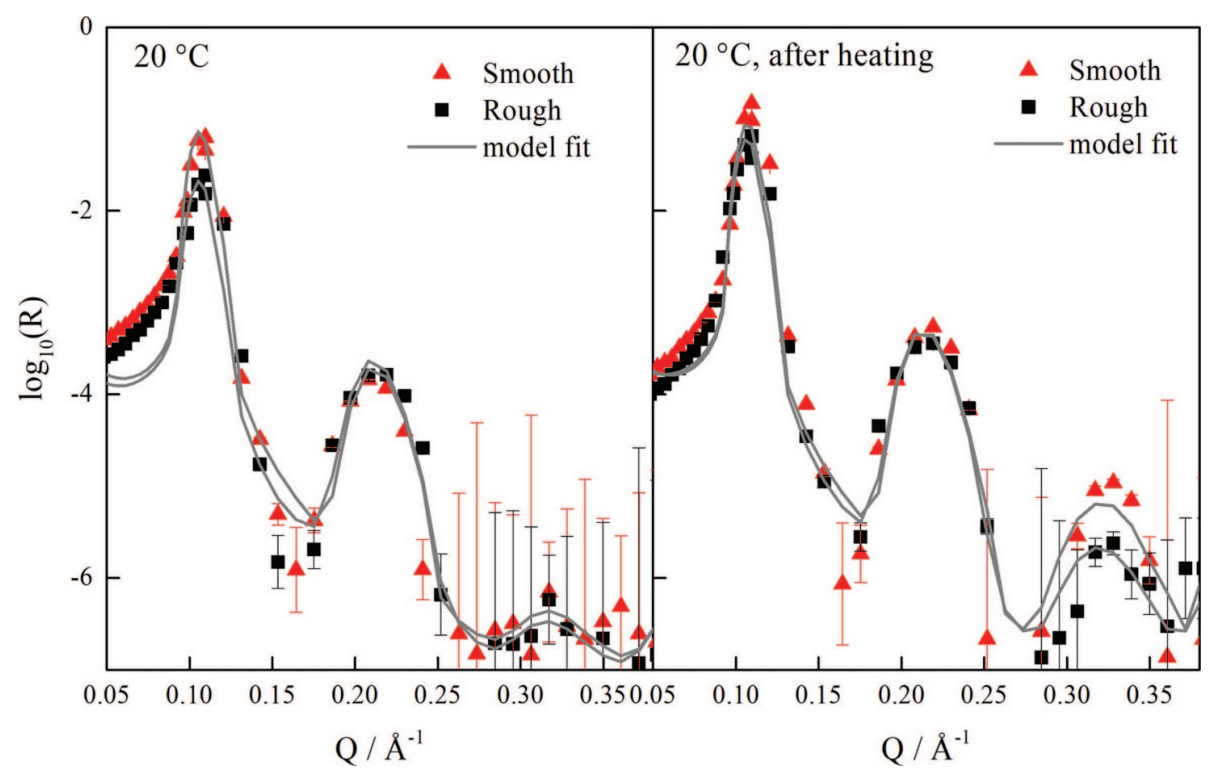

Fig. 2. Reflectivity data from Brij L4 against the smooth surface (triangles) and the rough surface (squares) after sample injection at $20^{\circ} \mathrm{C}$ on the left, and after heating the sample at $20^{\circ} \mathrm{C}$, on the right side.

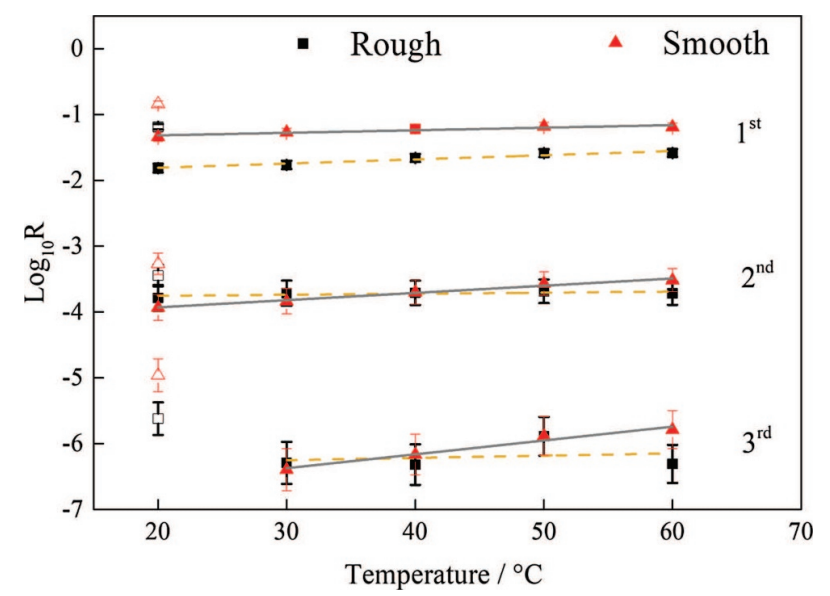

Fig. 3. Changes in the absolute reflectivity of the different order Bragg peaks with temperature, for the sample at the smooth surface (triangles) and the rough surface (squares). Open symbols indicate the values for the previously heated sample at $20^{\circ} \mathrm{C}$.

(left side of Fig. 2), the number of the lamellar sheets parallel to the surface at the smooth surface was found to be approximately 100 whereas that for the rough surface is around 30 repetitions. This shows that roughness even smaller than the lamellar spacing can disturb the layering and structure of self-assembled lamellae.

The effect of temperature change on the intensity of the different order Bragg peaks is shown in Figure 3. It is evident that the intensity of peaks increases with temperature for both surfaces and that the increase is more pronounced for the sample in contact with the smooth surface (larger gradient). The parameters that describe the fits to the reflectivity curves at the various temperatures are shown in 


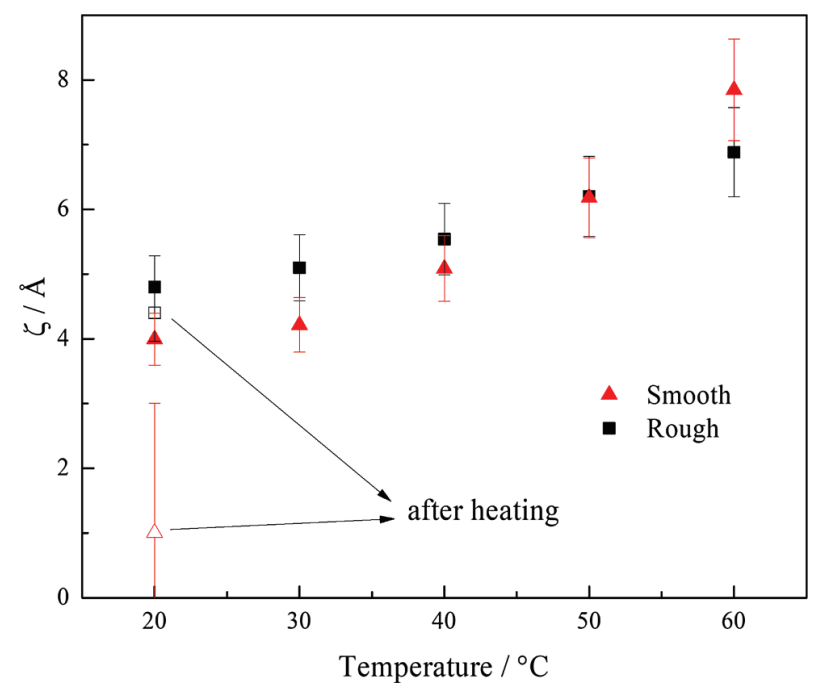

Fig. 4. Changes in thermal fluctuations, determined as the displacement of the layer described by a Debye-Waller factor, with temperature obtained from modelling reflectivity data for the smooth (triangles) and rough (squares) surfaces. The open symbols indicate the values after heating and then cooling the structure.

the supporting information (Tab. S1). The disorder, which is approximated as a root mean square displacement, increases with temperature but the other changes are small. The number of water molecules per surfactant molecule remains in the range 6 to 8. A significant effect was found to be the increase in the number of layers $(\mathrm{N})$ that are aligned at the interface. Our results show that increasing the temperature helps the lamellar structure to order and align particularly for the case of the smooth surface. As a result of increasing the temperature, thermal fluctuations in the structure play an important role in changing the relative intensity of the peaks. The mean displacement of lamellae due to thermal fluctuations obtained from the model fits at each temperature is shown in Figure 4. The amplitude of the thermal fluctuations that are reported in Figure 4 are of the same order as the roughness at the smooth surface. For the rough surface the local static variations in height are significantly larger than the thermal fluctuations and are seen to reduce substantially the orientational alignment. Some further test measurements on a surface with at least $100 \AA$ roughness did not show significant specular scattering but only the diffraction from bulk lamellar phase with poor alignment. The data for the sample did not allow further quantitative evaluation. These results correlate with observations of Imai et al. that large spherical particles completely destroy lamellar order in the similar system of $\mathrm{C}_{12} \mathrm{EO}_{5}$ /water [21].

The Caillé parameter for the lamellae calculated from equation 6 was found to increase from 0.04 at $20^{\circ} \mathrm{C}$ to 0.18 at $60^{\circ} \mathrm{C}$. This can be compared, for example, to values of 0.25 and 0.15 reported respectively for AOT/water and didodecyl dimethyl ammonium bromide (DDAB)/water systems at room temperature [50], or 0.08 reported for lipid bilayers formed from DMPC (1,2-dimyristoyl-sn-glycero-3phosphocholine) at $30^{\circ} \mathrm{C}$ [42]. From the Caillé parameter and using equation 5 , the product of the bending and compression moduli can be calculated. This suggests that the surfactant lamellae have broadly similar elastic moduli to those of saturated phospholipid membranes. Another parameter that might be used to distinguish between the two moduli, is the smectic penetration depth, $\Lambda$, which is the square root of the 
ratio of the bending modulus, $K$, and the compression modulus, $B$, of the membrane [23]:

$$
\Lambda=\sqrt{\frac{K}{B}} .
$$

As explained previously by Salditt et al. [51], for the case of a well-ordered multilayer where a small smectic penetration depth is expected, $\Lambda$ can be derived from careful analysis of off-specular and diffuse scattering data. In order to do that, high resolution scattering data in the lateral direction are required which were not accessible in the present experiment. Further, Broton et al. [23] have shown that scattering from multilayers with smectic penetration lengths that are several orders of magnitude different, vary most noticeably in higher $Q$. For our sample, the measured signal in that region is dominated by the background. Consequently, the experimental results and analysis in the present study, have primarily focused on interpretation of the disorder caused by the surface roughness on the structure of layers in the direction perpendicular to the interface.

The displacement of the surfactant layers increased as expected with temperature [49] in a similar way for Brij against both surfaces. However, when the sample is cooled again to $20^{\circ} \mathrm{C}$, after heating, the displacement for the Brij against the smooth surface is observed to be very small or even negligible. It is smaller than that seen after the initial injection of the sample at $20^{\circ} \mathrm{C}$. An explanation could be that when the sample was injected, part of the structure was dominated by the influence of flow on the orientation. The effect of shear flow on decreasing the ordering and alignment of lamellar structures has been shown previously for an AOT system [52]. Possibly such an alignment could have caused extra fluctuations that remain "frozen" and which could not be distinguished from the thermal fluctuations. While increasing temperature increases the fluctuations, the annealing allows the surfactants to align better with the surface. For the Brij at the rough surface, however, roughness distortion was strong enough to prevent further improvement of the alignment. Another explanation could be that the packing of surfactant molecules changes during the annealing process. It has been observed previously that in the case of alkyl-aryl-ethylene oxide amphiphiles, the ethylene oxide chains are aligned normal to the surface of the layers, and the alkyl chains are tilted $25^{\circ}$ relative to the surface normal at room temperature, however when the sample is annealed at higher temperatures, both tails align themselves normal to the interface [53]. Detailed studies are required to understand structural changes occurring during the thermal annealing process.

Annealing the sample showed a significant increase in the intensity of the diffraction, in particular the higher order Bragg peaks. The third order peak could be seen when the temperature was increased, indicating that the number of layers aligned with the planar surface increased. Annealing showed a stronger effect on the sample at the smooth surface compared to the rough one. The number of the aligned layers at the smooth surface increased to at least 120 layers whereas that at the rough surface was about 70 layers. Note that 120 bilayers correspond to a thickness of approximately $0.7 \mu \mathrm{m}$ which for a typical reflectometry experiment is approximately same as the coherency length of the beam in $z$ direction. This suggests that the number of layers may be larger than 120 but adding them do not make a significant change in the intensity to the measured signal.

The parameters obtained from modeling reflectivity data have uncertainties that arise from both data reduction and analysis. However, the uncertainty, for example in the number of layers can affect the results up to $20 \%$ but does not explain the significant difference for the same sample against the two different surfaces. Parameters such as number of stacked bilayer and the hydration of the layers are strongly affected by the relative intensity of the various different order Bragg peaks where 
a)
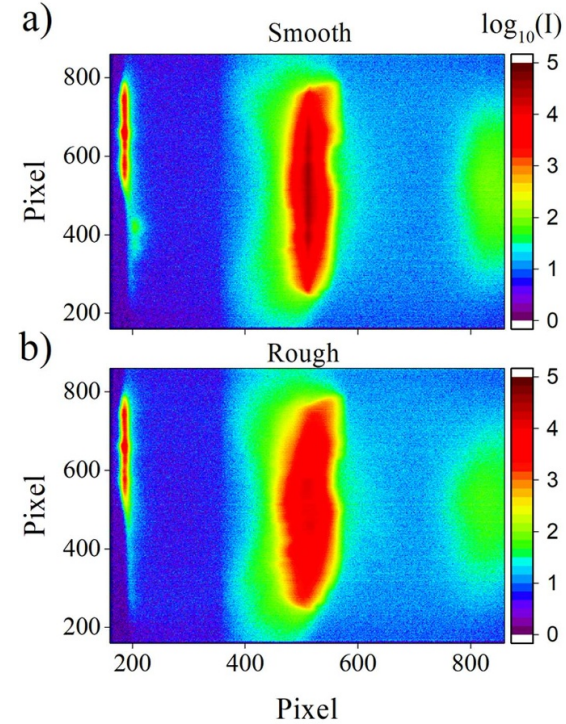

c)

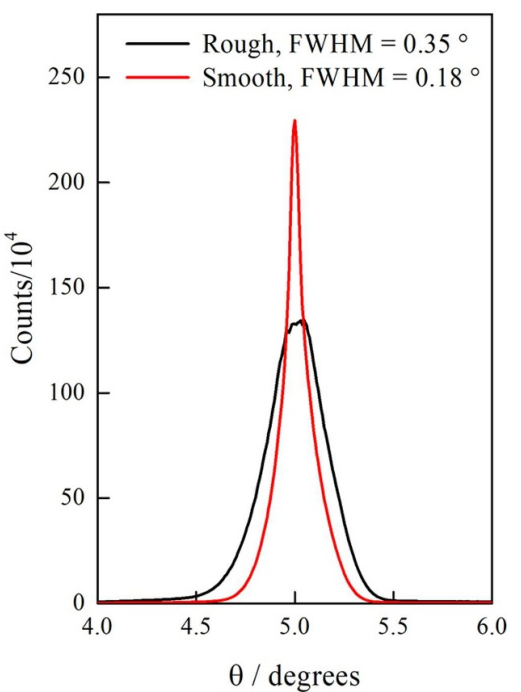

Fig. 5. Scattering pattern observed on the two-dimensional detector when the first order Bragg peak is in the specular condition ( $\mathrm{a}$ and $\mathrm{b}$ ) and the sum of the intensity in vertical lines down the detector (c) showing the different FWHM. The sample corresponds to 55 vol.\% Brij L4 in $\mathrm{D}_{2} \mathrm{O}$. Data are shown for the sample after heating when measured at $20^{\circ} \mathrm{C}$.

distinguishing the true reflectivity data from the background is relatively straightforward.

One of the evident differences between the reflectivity from the sample at two surfaces was the intensity of the Bragg peaks. Figures 5a and 5b show the twodimensional shape of the first order Bragg peaks when the peak is at the specular reflection condition, after heating and then cooling the sample. The sum along the vertical direction for each of the detector image is shown in Figure 5c. The first order Bragg peak from the sample at the rough surface is less intense and more diffuse. The peak has nearly twice the width, which indicates again that layers of surfactant are less well-aligned with the interface. This was consistent with the results obtained from the model fits that there are nearly twice as many layers aligned with the smooth surface at as compared to the rough surface. The grain size obtained from the width of the first order Bragg peak at specular condition using equation (7) showed that the number of layers parallel to the surface is the same order as that obtained from fitting the full reflectivity curve (i.e. over 100 layers for the sample against the smooth surface and about 70 for the sample against the rough surface.).

The crystallite size of lamellae that were not aligned parallel to the substrate was estimated using equation (7) for the diffraction data measured in the off-specular condition and the results are shown in Figure S8, supporting information. For the sample that was against the smooth surface, the grain or crystal size corresponded to 25 layers whereas for the sample at rough surface it was about 17 layers. This suggests, given the calculated penetration of $10 \mathrm{~s}$ of $\mu \mathrm{m}$ that the influence of the interface can extend deep into the bulk of the sample altering the size of even randomly oriented crystallites. However, part of the initial alignment and domain size may arise from the flow that occurs on filling the cell. This is likely to be different for the two surfaces.

The overall description of the structure aligned with the surface and aligned in other directions, is confirmed by looking at the width of the first order peak from the lamellar structure as the sample is scanned in angle. This is shown in Figure 6. The 


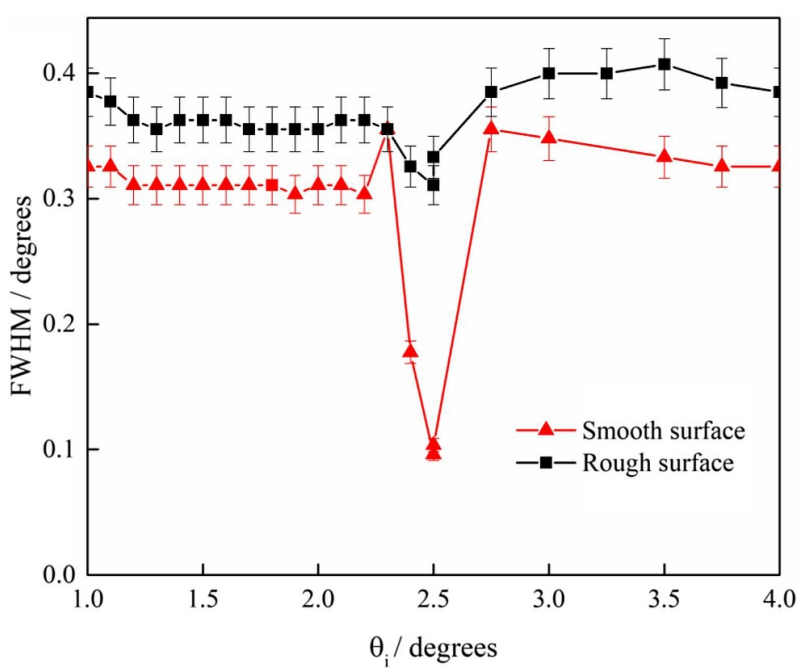

Fig. 6. Width of the 1 st order diffraction peak for 55 vol.\% Brij $\mathrm{L} 4$ in $\mathrm{D}_{2} \mathrm{O}$ after heating measured at $20^{\circ} \mathrm{C}$ as a function of sample orientation with respect to the incident beam, $\theta_{\mathrm{i}}$. When the diffraction peak is at the specular condition marked narrowing occurs as there are many well-aligned layers at the interface, particularly for the smooth surface. Note that the specular reflection occurs at $\theta_{\mathrm{i}}=\theta_{\mathrm{f}}=2.5$ degrees.

peak is seen on the detector for a range of angles either side of the condition where it is aligned with the specular reflection. On either side of the specular condition the peak is broad. It becomes markedly narrower at the specular reflection. At the specular condition, the peak width is much narrower although the effect is more pronounced for the smooth surface. The different domain size found for randomly oriented lamellae, is again indicative of the surface or the flow altering the overall structural morphology when the cell is filled.

The rocking scans measured at the highest temperature and after cooling the sample back to $20^{\circ} \mathrm{C}$ are shown in Figure 7 . There is a significant difference at each temperature between the rocking curves measured for the sample against the two surfaces. Rocking scans for the sample are sharper and more intense when the sample is adjacent to the smooth surface, which confirms the results found from the model fits that the lamellar forms a better-aligned structure at that interface.

The intensity of the peaks after heating and subsequent cooling for the sample are increased compared to those measured at $60^{\circ} \mathrm{C}$ for both surfaces. This increase was however more pronounced for the sample at the smooth surface. This shows that annealing effect can improve the alignment at the smooth surface more than that at the rough surface.

The effects of surface roughness and the temperature were similar on the intensity and the width of the reflectivity peaks as well as for the rocking curves measured for a sample of $40 \mathrm{vol} . \%$ Brij in $\mathrm{D}_{2} \mathrm{O}$ against the same smooth and rough surfaces (see Figs. S4-S7, supporting information). However, modelling the data and obtaining quantitative values for the fit parameters was more challenging than for the more concentrated system. The lamellar spacing becomes larger and the different order Bragg peaks are close to each other. Hence the effects of overlapping peaks are more pronounced. This means that estimating reflectivity and subtracting the true background from overlapping peaks becomes very challenging.

As previously, the strong Bragg peaks indicate clearly a layered structure. The position of the first order Bragg peak corresponds to a repeat distance of $84 \AA$ for the 


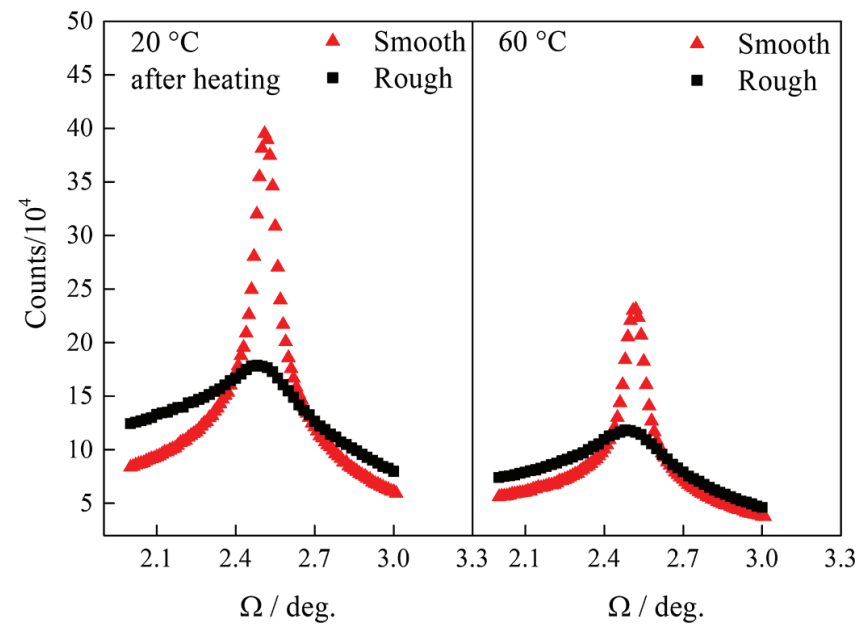

Fig. 7. Rocking curves measured on the first-order Bragg peak for 55 vol.\% Brij L4 in $\mathrm{D}_{2} \mathrm{O}$ at the highest temperature and after heating. $\Omega$ is the angle of the sample.

bilayers. For a dilution from 55 vol. $\%$ to 40 vol.\% surfactant, the spacing is expected to change from $58 \AA$ to $80 \AA$. This correspondence is close enough to suggest that the lamellar structure at the interface corresponds to the bulk concentration.

The reflection peak that is shown in Figure 5 is apparently on a background that is part of a Debye-Scherrer ring of diffracted intensity from the bulk that is partially aligned. The slit collimation used in this experiment and the relatively small part of the total scattering that is observable on the two-dimensional detector precludes quantitative determination of the orientation distribution. The curvature of this background is also apparent in the profiles obtained by summing vertically that are shown in Figure S3 with a tail of higher intensity on the low-angle side of the diffraction peak. The other apparent texture in the observed peaks probably arises from the non-uniform distribution of neutron flux in the guide system of the reflectometer but this spatial variation is not significant in respect of the analysis of integrated intensities that has been described above. A more advanced data analysis that would include these features, might allow further information to be obtained from the background scattering.

\section{Conclusions}

The well-aligned multilayer structure formed at a solid/liquid interface allows better determination of Bragg peak intensity than experiments on randomly aligned lamellar aggregates as seen in conventional small-angle neutron scattering such as those of Strey et al. [32]. The present study has exploited this advantage to explore the effect of surface roughness and temperature on the alignment of the non-ionic surfactant, $\mathrm{C}_{12} \mathrm{EO}_{4}$, at solid surfaces using neutron reflectometry and small-angle scattering. The results showed that 55 vol. $\% \mathrm{C}_{12} \mathrm{EO}_{4}$ in $\mathrm{D}_{2} \mathrm{O}$ self-assembles into multilayer layer structures aligned with the surface that extends about $1 \mu \mathrm{m}$ into the bulk. Such a system provides a model for understanding physics of multilayers. Roughness of the order of the lamellar spacing reduces, but did not completely eliminate the alignment of the lamellar structure at the interface. At a smooth surface, heating the sample and subsequent cooling back to $20^{\circ} \mathrm{C}$ increases the number of aligned bilayers from 100 to 120 layers. For the rough surface this increase was from 30 to 70 layers. 
In general fluctuations determined from the reflectivity were found to increase as expected with temperature. The reversibility was found to be different for the sample near smooth and rough surfaces, as it is apparent that near the smooth surface some static disorder was present in the initial sample. A possible explanation could be that when the sample was measured at $20^{\circ} \mathrm{C}$ immediately after injection, the alignment was influenced by the flow orientation. When the sample is heated, the larger fluctuations can allow the sample to align with the interface.

The major features of the orientation and structure are found also at the lower surfactant concentration of $40 \mathrm{vol} . \%$. This implies that structures tailored with particular lamellar spacings can be obtained simply by concentration change. It is interesting that very strong diffraction can be obtained from fluid samples, reaching more than 0.1 in reflectivity. The width of the Bragg diffraction peak can be modified by simple thermal treatment. Such a sample provides an ideal model system to study different types of disorder in the self-assembled structures and to explore the advantages and/or limitations of technique.

Open access funding provided by Uppsala University.

Open Access This is an open access article distributed under the terms of the Creative Commons Attribution License (https://creativecommons.org/licenses/by/4.0/), which permits unrestricted use, distribution, and reproduction in any medium, provided the original work is properly cited.

Publisher's Note The EPJ Publishers remain neutral with regard to jurisdictional claims in published maps and institutional affiliations.

\section{References}

1. J. Yang. Curr. Opin. Colloid. Interface Sci. 7, 276 (2002)

2. C. Prieto, L. Calvo, J. App. Chem. 2013, 930356 (2013)

3. E. Ha, W. Wang, J.Y. Wang, J. Pharm. Sci. 91, 2252 (2002)

4. B.A. Kerwin, J. Pharm. Sci. 97, 2924 (2008)

5. J. Penfold, I. Tucker, R.K. Thomas, Langmuir 21, 6330 (2005)

6. G.D. Parfitt, C.H. Rochester, Adsorption from Solution at the Solid/Liquid Interface (Academic Press London, New York, 1983)

7. J.N. Israelachvili, Intermolecular and Surface Forces (Academic Press, 2008)

8. R. Nagarajan, Langmuir 18, 31 (2001)

9. G.T. Pickett, T.A. Witten, S.R. Nagel, Macromolecules 26, 3194 (1993)

10. Z.G. Workineh, A.G. Vanakaras, Polymers 6, 2082 (2014)

11. M.I. Boamfa, M.W. Kim, J.C. Maan, Th. Rasing, Nature 421, 149 (2003)

12. M.C. Gerstenberg, J.S. Pedersen, G.S. Smith, Phys. Rev. E 58, 8028 (1998)

13. N.A. Lockwood, J.K. Gupta, N.L. Abbott, Surf. Sci. Rep. 63, 255 (2008)

14. L.S. Hirst, G. Charras, Nature 544, 164 (2017)

15. L. Ginsberg, J.R. Atack, S.I. Rapoport, N.L. Gershfeld, Mol. Chem. Neuropathol. 19, 37 (1993)

16. S. Jeong, J.Y. Kim, B.H. Kim, H. Moon, S.O. Kim, Mater. Today 16, 468 (2013)

17. V. Kapaklis, S. Grammatikopoulos, R. Sordan, A. Miranda, F. Traversi, H. von Kanei, D. Trachylis, P. Poulopoulos, C. Politis, J. Nanosci. Nanotechnol. 10, 6056 (2010)

18. L. Chen, S. Lin, C. Huang, E. Chen, Colloids Surf. A 135, 175 (1998)

19. N. Li, R.K. Thomas, A.R. Rennie, J. Colloid Interface Sci. 369, 287 (2012)

20. M. Imai, R. Mawatari, K. Nakaya, S. Komura, Eur. Phys. J. E 13, 391 (2004)

21. M. Imai, Y. Suganuma, K. Nakaya, S. Komura, J. Phys.: Condens. Matter 17, S2929 (2005) 
22. Y. Suganuma, N. Urakami, R. Mawatari, S. Komura, K. Nakaya-Yaegashi, M. Imai, J. Chem. Phys. 129, 134903 (2008)

23. G. Brotons, T. Salditt, M. Dubois, Th. Zemb, Langmuir 19, 8235 (2003)

24. N. Prinz, T. Nylander, Nano Lett. 14, 4286 (2014)

25. S. Wu, L. Shi, L.B. Garfield, R.F. Tabor, A. Striolo, B.P. Grady, Langmuir 21, 6091 (2011)

26. G. Fragneto, R.K. Thomas, A.R. Rennie, J. Penfold, Langmuir 12, 6036 (1996)

27. S. Nouhi, M.S. Hellsing, V. Kapaklis, A.R. Rennie, J. Appl. Cryst. 50, 1066 (2017)

28. S. Mattauch, A. Koutsioubas, S. Pütter, J. Large-scale Res. Facil. 1, A8 (2015)

29. S. Mattauch, A. Koutsioubas, U. Rücker, D. Korolkov, V. Fracassi, J. Daemen, R. Schmitz, K. Bussmann, F. Suxdorf, M. Wagener, P. Kämmerling, H. Kleines, L. Fleischhauer-Fuß, M. Bednareck, V. Ossoviy, A. Nebel, P. Stronciwilk, S. Staringer, M. Gödel, A. Richter, H. Kusche, T. Kohnke, A. Ioffe, E. Babcock, Z. Salhi, T. Bruckel, J. Appl. Cryst. 51, 646 (2018)

30. D.J. Mitchell, G.J.T. Tiddy, L. Waring, T. Bostock, M.P. McDonald, J. Chem. Soc. Faraday Trans. I 79, 975 (1983)

31. P.G. Nilsson, H. Wennerström, B. Lindman, J. Phys. Chem. 87, 1377 (1983)

32. R. Strey, R. Schomäcker, D. Roux, F. Nallet, U. Olsson, J. Chem. Soc. Faraday Trans. 86, 2253 (1990)

33. S. Nouhi, M. Pascual, M.S. Hellsing, H.M. Kwaambwa, M.W.A. Skoda, F. Höök, A.R. Rennie, Colloids Surf. B 168, 68 (2018)

34. F. Abelès, J. Phys. Radium 11, 307 (1950)

35. F. Abelès, J. Phys. Radium 11, 310 (1950)

36. A.R. Rennie, Neutron Reflection and Neutron Reflectometers, Analysis Programs (2018), http://www.reflectometry.net/reflect.htm\#Analysis

37. M.S. Hellsing, A.V. Hughes, A.R. Rennie, Langmuir 27, 4669 (2011)

38. L. Névot, P. Croce, Rev. Phys. Appl. 15, 761 (1980)

39. G.L. Squires, Introduction to Thermal Neutron Scattering (Dover, Mineola, NY, 1996)

40. B.E. Warren, X-Ray Diffraction (Addison-Wesley, Reading, MA, 1969)

41. A. Caillé, C. R. Acad. Sci. Paris Série B 274, 891 (1972)

42. J.F. Nagle, S. Tristram-Nagle, Biochim. Biophys. Acta (BBA) - Rev. Biomembranes 1469, 159 (2000)

43. M. Rappolt, G. Pabst, in Structure and Dynamics of Membranous Interfaces edited by K. Nag (John Wiley \& Sons, Hoboken NJ, 2008), Chapter 3: Flexibility and Structure of Fluid Bilayer Interfaces, pp. 45-81

44. S.J.S. Qazi, A.R. Rennie, J.K. Cockcroft, M. Vickers, J. Colloid Interface Sci. 338, 105 (2009)

45. A.R. Rennie, M.S. Hellsing, E. Lindholm, A. Olsson, Rev. Sci. Inst. 86, 016115 (2015)

46. M. Wolff, P. Kuhns, G. Liesche, J.F. Ankner, J.F. Browning, P. Gutfreund, J. Appl. Cryst. 46, 1729 (2013)

47. Z.X. Li, A. Weller, R.K. Thomas, A.R. Rennie, J.R.P. Webster, J. Penfold, R.K. Heenan, R. Cubitt, J. Phys. Chem. B 103, 10800 (1999)

48. Z.X. Li, J.R. Lu, R.K. Thomas, A. Weller, J. Penfold, J.R.P. Webster, D.S. Sivia, A.R. Rennie, Langmuir 17, 5858 (2001)

49. C. Loison, M. Mareschal, K. Kremer, F. Schmid, J. Chem. Phys. 119, 13138 (2003)

50. F. Nallet, R. Laversanne, D. Roux, J. Phys. II France 3, 487 (1993)

51. T. Salditt, C. Munster, U. Mennicke, C. Ollinger, G. Fragneto, Langmuir 19, 7703 (2003)

52. R.J.L. Welbourn, F. Bartholomew, P. Gutfreund, S.M. Clarke, Langmuir 33, 5982 (2017)

53. G. Liu, G.L. Baker, Soft. Matter 4, 1094 (2008) 